\title{
Multiple Sclerosis Patients have an Altered Gut Mycobiome and Increased Fungal to Bacterial Richness Running Title: RRMS Patients have Gut Fungal Dysbiosis
}

\author{
Meeta Yadavi,2,\#, Soham Ali3,\#, Rachel L. Shrode, Shailesh K. Shahi ${ }^{1}$, Samantha N. \\ Jensen ${ }^{1,5}$, Jemmie Hoang ${ }^{6}$, Samuel Cassidy $^{8}$, Heena Olalde ${ }^{8}$, Natalya Guseva ${ }^{1}$, \\ Mishelle Paullus ${ }^{8}$, Catherine Cherwin ${ }^{6}$, Kai Wang ${ }^{7}$, Tracey Cho ${ }^{8}$, John Kamholz ${ }^{8}$, \\ Ashutosh K. Mangalam ${ }^{1,4,5,9 *}$ \\ 1Department of Pathology, Carver College of Medicine, University of lowa, lowa City, IA, USA, \\ 2University of lowa College of Dentistry, lowa City, IA, USA, ${ }^{3}$ Carver College of Medicine, University \\ of lowa, lowa City, IA, USA, ${ }^{4}$ Informatics Graduate Program, University of lowa, lowa City, IA, USA, \\ ${ }^{5}$ Interdisciplinary Graduate Program in Immunology, University of lowa, lowa City, IA, USA, ${ }^{6}$ College \\ of Nursing University of lowa, lowa City, IA, USA, ${ }^{7}$ Department of Biostatistics, College of Public \\ Health, University of lowa, lowa City, IA, USA ${ }^{8}$ Department of Neurology, University of lowa \\ Hospitals and Clinics, lowa City, IA, USA, and ${ }^{9}$ lowa City VA Health System, lowa City, IA, USA.
}

\section{${ }^{*}$ Corresponding author:}

Dr. Ashutosh K Mangalam

Department of Pathology, University of lowa Carver College of Medicine 25 S. Grand Avenue, $1080 \mathrm{ML}$ lowa City, IA 52242, USA

Phone: 1-319-335-8558

Email: Ashutosh-mangalam@uiowa.edu \# Authors contributed equally 


\section{Abstract}

Trillions of microbes such as bacteria, fungi, and viruses exist in the healthy human gut microbiome. Although gut bacterial dysbiosis has been extensively studied in multiple sclerosis (MS), the significance of the fungal microbiome (mycobiome) is an understudied and neglected part of the intestinal microbiome in MS. The aim of this study was to characterize the gut mycobiome of patients with relapsing-remitting multiple sclerosis (RRMS), compare it to healthy controls, and examine its association with changes in the bacterial microbiome. We characterized and compared the mycobiome of 20 RRMS patients and 33 healthy controls $(\mathrm{HC})$ using Internal Transcribed Spacer 2 (ITS2) and compared mycobiome interactions with the bacterial microbiome using $16 \mathrm{~S}$ rRNA sequencing. Our results demonstrate an altered mycobiome in RRMS patients compared with HC. RRMS patients showed an increased abundance of Basidiomycota and decreased Ascomycota at the phylum level with an increased abundance of Candida and Epicoccum genera along with a decreased abundance of Saccharomyces compared to HC. We also observed an increased ITS2/16S ratio, altered fungal and bacterial associations, and altered fungal functional profiles in MS patients compared to HC.

This study demonstrates that RRMS patients had a distinct mycobiome with associated changes in the bacterial microbiome compared to $\mathrm{HC}$. There is an increased fungal to bacterial ratio as well as more diverse fungal-bacterial interactions in RRMS patients compared to HC. Our study is the first step towards future studies in 
delineating the mechanisms through which the fungal microbiome can influence MS disease.

\section{Introduction}

Multiple Sclerosis (MS) is a neuroinflammatory autoimmune disease that affects $\sim 2.5$ million people worldwide. Though MS has different clinical subtypes, most of the patients ( 85\%) present with relapsing-remitting MS (RRMS). The precise etiopathogenesis of MS is unclear, but both genetic and environmental factors have been suggested to play an important role in susceptibility and the pathogenesis of MS. In recent years multiple groups, including ours, have highlighted the role of gut bacteria in the pathobiology of $\mathrm{MS}^{1-8}$, and it has emerged as an important environmental factor. However, the gut microbiome consists of non-bacterial microbes such as fungi, viruses, and archaea ${ }^{9,10}$, yet the role of the fungal microbiome (mycobiome) is not well studied in MS.

Although fungi make up approximately $0.1 \%$ of the gastrointestinal tract ${ }^{10,11}$, their impact on human health is significant due to their ability to regulate local and systemic host immune response ${ }^{12,13}$. The importance of the fungal microbiome on the immune system was demonstrated in a study where mice that were rewilded had increased intestinal abundance of fungi along with an expansion of granulocytes compared to laboratory mouse controls ${ }^{12}$. While in human peripheral blood $50-70 \%$ cells are neutrophils, only $10-25 \%$ cells are neutrophils in mouse peripheral blood ${ }^{14}$. Additionally, an oral antifungal treatment of mice in colitis model showed increased disease severity and exacerbated allergic airway disease with increased Aspergillus, Wallemia, and Epiccocum and decreased Candia spp. Moreover, oral administration of a mixture of these three fungi (Aspergillus amstelodami, Epicoccum nigrum, and 
Wallemia sebi) was sufficient to recapitulate the exacerbating effects of antifungal drugs on allergic airway disease ${ }^{13}$. The importance of the mycobiome in human health and disease is validated by studies showing alterations in mycobiome composition in various autoimmune diseases such as inflammatory bowel disorders (IBD), ankylosing spondylitis (AS), and type 2 diabetes mellitus (DM) compared to healthy controls $(\mathrm{HC})^{15-17}$. These studies have also shown correlations between bacterial and fungal microbiota that are altered in disease states. The relationship between gut bacteria and fungi has been demonstrated in the murine model where bacteria outnumbered fungi by greater than three times prior to antibiotic administration, but after antibiotic administration bacterial abundance dropped three-fold while fungal abundance increased around 40 -fold ${ }^{18}$. Thus all these observations suggest that gut mycobiome might play an important role in the health and disease including MS.

Based on the association of gut mycobiota in other autoimmune diseases and on the described relationship between gut bacteria and MS, we hypothesized that the gut fungal microbiota might be altered in MS patients. Therefore, in this study we profiled the fungal microbiota and associated fungal functional characteristics in RRMS patients (MS) $(n=20)$ and compared them with those of $\mathrm{HC}(n=33)$. We also analyzed the bacterial microbiome of the same patients to determine the correlation between the fungal and bacterial microbiome. We observed that the MS patients have a distinct fungal microbiome compared to $\mathrm{HC}$, with differential abundances of multiple fungal genera and fungal functions as well as altered fungal-bacterial relationships. A distinct fungal microbiome in RRMS patients could have implications in the pathogenesis of MS, potentially through functional differences and altered interactions with the bacterial microbiome. 


\section{Methods}

\section{Standard Protocol Approvals, Registrations, and Patient Consents}

The study was done in accordance with the guidelines approved by the University of lowa Institutional Review Board (IRB). A prior written informed consent was obtained from all the subjects to participate in the study.

\section{Human Subjects Enrollment}

Relapsing-Remitting Multiple Sclerosis (RRMS) patients $(n=22)$ who fulfill McDonald diagnostic criteria for MS were recruited from the Neuroimmunology Clinic at the University of lowa Hospital Clinics (UIHC), at the University of lowa. Healthy controls $(\mathrm{HC})(\mathrm{n}=34)$ were also recruited through the University of lowa (Table 1). Individuals eligible for the study were those 18-63 years of age with a diagnosis of RRMS. All patients were either on MS treatment or not on treatment for at least three months prior to enrollment. Patients and healthy controls were asked to provide samples at least four weeks after their last dose of oral antibiotics or laxatives, and at least three months after their last colonoscopy. Those with active pregnancy or a history of bariatric surgery were excluded from the study.

Table 1. Biometric and MS Patient Treatment Data

\begin{tabular}{|l|c|c|c|}
\hline & HC & MS & p-value \\
\hline Age: Mean \pm SD & $42 \pm 14$ & $45 \pm 7.8$ & t-test: 0.33 \\
\hline Sex: Male / Female & $5 / 28$ & $5 / 15$ & Fisher: 0.48 \\
\hline BMI: Mean \pm SD & $24 \pm 3.7$ & $30 \pm 7.9$ & t-test: 0.015 \\
\hline
\end{tabular}




\begin{tabular}{|l|c|c|l|}
\hline Treatment: Yes / No & NA & $16 / 4$ & NA \\
\hline Treatment & & & \\
\hline Interferon beta & & 3 & \\
\hline Glatiramer acetate & & 4 & \\
\hline Ocrelizumab & & 3 & \\
\hline Dimethyl fumarate & & 6 & \\
\hline Not on treatment & & 4 & \\
\hline
\end{tabular}

\section{Human Sample Collection}

Stool samples were collected by patients and HC in Commode Specimen Collection kits (Fisher PA, USA) provided to them by our laboratory along with the instruction sheet and shipped on frozen gel packs to our laboratory by overnight delivery ${ }^{2}$. Once in the laboratory, the stool was aliquoted and stored at -80 degrees until DNA extraction. The same collection kits, instruction sheets, and processing methods were used for fecal samples from MS patients and HC.

\section{DNA Extraction and Sequencing}

Microbial DNA was extracted from each fecal sample using Qiagen DNeasy PowerLyser PowerSoil Kit (Qiagen, Germantown, MD) per the manufacturer's instructions using a bead-beating step (PowerLyzer 24 Homogenizer, Omni International, USA). The fungal microbiome was analyzed using Two-step PCR as described previously for bacterial microbiome analysis ${ }^{19}$ except instead of $16 \mathrm{~S}$ rRNA; we targeted the internal transcribed spacer 2 (ITS2) region of fungal 18S rRNA using 
the following primers: forward primer 86 F(5'-3') GTGAATCATCGAATCTTTGAA and reverse primer 4R (5'-3') TCCTCCGCTTATTGATATGC 20 . Fungal PCR conditions were as follows: $95^{\circ} \mathrm{C}$ for $5 \mathrm{~min}, 35$ cycles of $95^{\circ} \mathrm{C}$ for $30 \mathrm{~s}, 59^{\circ} \mathrm{C}$ for $30 \mathrm{~s}, 72^{\circ} \mathrm{C}$ for $30 \mathrm{~s}$, and $72^{\circ} \mathrm{C}$ for $10 \mathrm{~min}$. Bacterial $16 \mathrm{~S}$ rRNA sequencing was performed as per protocol published by our lab ${ }^{19}$.

\section{Metagenomic Profiling}

The raw fungal ITS2 (internal transcribed spacer 2) sequence data was processed utilizing DADA2 ${ }^{21}$. In brief, the reads were trimmed to remove the primer sequences, then truncated based on a quality score of 25 . Reads were then denoised to infer the exact sequence variants by resolving single-nucleotide errors. Paired forward and reverse reads were merged, and any resulting chimeras were removed to produce amplicon sequence variants (ASVs). These ASVs were then assigned fungal taxonomy at the genus level using a naive Bayesian classifier method and the UNITE database (Version 8.2) ${ }^{22}$. After the removal of three samples (2 MS and one $\mathrm{HC}$ ) with less than 1000 reads, 20 MS samples and 33 HC samples were available for fungal profiling, with a median number of reads of 37,331 .

The raw sequence data of the V3-V4 region of bacterial 16S rRNA was processed utilizing DADA2. These sequences were processed as described above for ITS sequences, and the final ASVs were assigned bacterial taxonomy at the kingdom to species levels using the Silva database (version 138.1$)^{23}$ with a median number of reads of 49,976 .

\section{Functional Profiling}

The functional profile of the gut mycobiome was generated using FungalTraits 
database $^{24}$. Of the 142 identified fungal genera in this study, 86 had functional entries in this database, and the functional profile of each sample was then inferred using its fungal composition and the functional data of each of these 86 fungal genera.

\section{Statistical Analysis}

Analysis and figure generation of alpha diversity, beta diversity, and differential abundance of all features was performed entirely in $\mathrm{R}$ (Version 4.0.3) using the vegan ${ }^{25}$ and ggpubr ${ }^{26}$ packages with custom $\mathrm{R}$ scripts. Prior to statistical analysis, the fungal and bacterial datasets were each constant-sum scaled to one million reads and generalized log-transformed. The fungal and bacterial data were then filtered to remove low prevalence taxa. Alpha diversity and differential abundance analysis between the MS and $\mathrm{HC}$ groups were performed using the Wilcoxon test, and p-values were adjusted using the Benjamini-Hochberg algorithm. PERMANOVA was performed to analyze the statistical significance of beta-diversity clustering.

Inter-kingdom correlation analysis was conducted between the bacteria and fungi at the genus level using Spearman's rank correlation, and only correlations showing p-value less than 0.05 are reported. Bootstrapped random forest analysis was conducted using the Boruta package ${ }^{27}$ at the suggested significance level of 0.01 with 500 trees for 100 iterations.

\section{Data Availability:}

The microbiota $16 \mathrm{~S}$ and ITS2 sequencing analysis data have been deposited to the Sequence Read Archive (https://www.ncbi.nlm.nih.gov/sra) under PRJNA732670 for free public access. All other data are available from the corresponding author upon request. 


\section{Results}

\section{Fungal microbiota of RRMS patients is different from healthy controls}

To characterize the fungal microbiome diversity, we sequenced fecal samples of 20 RRMS patients and 33 healthy controls $(\mathrm{HC})$ using ITS2 rRNA sequencing. A total of 136 fungal genera were identified, with 31 unique to the $\mathrm{HC}$ group, 73 unique to the MS group, and 32 genera were present in both groups. Alpha diversity using the Chao 1 index showed greater richness in MS than in $\mathrm{HC}$ (Fig 1A), though this was not statistically significant $(p>0.08)$. Principal coordinate analysis of beta diversity using Bray-Curtis dissimilarity (Fig 1B) demonstrated distinct clustering of the mycobiome of MS compared with $\mathrm{HC}(\mathrm{p}=0.004)$.

Overall, the two most prominent phyla by relative abundance were Ascomycota and Basidiomycota (Fig 1C), and the top five fungal genera were Saccharomyces, Candida, Malassezia, Penicillium, and Cladosporium (Fig 1D), comprising $75.5 \%$ of all the identified fungal genera in the samples.

Examining the differential abundance between the MS and $\mathrm{HC}$ groups at the phylum level showed a lower abundance of Ascomycota $\left(\mathrm{p}_{\mathrm{adj}}=0.011\right)$ and higher abundance of Basidiomycota $\left(\mathrm{p}_{\mathrm{adj}}=0.011\right)$ in the MS group compared to $\mathrm{HC}\left(\mathrm{p}_{\mathrm{adj}}=0.011\right.$ and 0.011 , respectively). Previous studies in IBD and AS have shown that there is a strong negative correlation between Basidiomycota and Ascomycota characterized by a higher Basidiomycota/Ascomycota ratio in $\mathrm{IBD}^{17}$ and $\mathrm{AS}^{16}$. In this study, we also observed that MS patients have an increased Basidiomycota/Ascomycota ratio ( $p=$ 0.0053) compared to HC (Fig 1E). Thus, our data demonstrates a phylum-level shift in MS towards Basidiomycota and away from Ascomycota. Interestingly, within the 
Ascomycota phylum, the genera Candida $\left(p_{a d j}=0.021\right)$ and Epicoccum $\left(p_{a d j}=0.021\right)$ were more abundant in the MS group (Fig 2A \& B), while only Saccharomyces $\left(p_{a d j}=\right.$ 0.021) was depleted in the MS group (Fig 2B) compared with HC. Species-level analysis showed Saccharomyces cerevisiae comprising $88 \%$ of the Saccharomyces genus and Candida albicans comprising $81 \%$ of the Candida genus across all samples. Thus, our study finds that MS patients have a distinct mycobiome compared to HC with enrichment or depletion of certain fungi.

\section{Random Forest Analysis to identify the potential discriminating fungi}

We next assessed the ability of the gut mycobiota profile to predict disease phenotype in our samples. A bootstrapped random forest algorithm of 500 trees was used to generate a predictive model based on the gut mycobiota profiles of the samples. The Boruta algorithm was then utilized to identify the fungal genera that were most important in distinguishing between MS and $\mathrm{HC}$ samples at a significance level of 0.01 . Interestingly, the four aforementioned genera, Candida, Saccharomyces, Epicoccum, and Malassezia, were again identified (Fig 2C). The Boruta algorithm additionally identified Penicillium which is decreased in MS, and Malassezia, which is increased in MS (Fig 2C), as a significantly important feature in distinguishing MS from HC. Thus, the random forest classification analysis suggests that these fungal taxa could be important discriminators between MS and $\mathrm{HC}$.

\section{Functional Profile of Gut Mycobiome in MS Patients}

Analysis of the mycobiome functional profile determined from the FungalTraits database revealed several enzymes whose enrichment differed significantly between MS and HC samples. Levels of amino acid permease $\left(p_{a d j}=0.029\right)$, cellobiohydrolase $\left(p_{a d j}=0.011\right)$, endoglucanase $\left(p_{a d j}=0.029\right)$, and invertase $\left(p_{a d j}=0.0211\right)$ were lower in MS patients 
(Fig 3). These results suggest potential differences in fungal metabolism and functional phenotypes in the gut of MS patients.

\section{Gut Bacterial Microbiome of RRMS patients is different from healthy}

\section{controls}

We also characterized the bacterial microbiome of the same samples using 16S rRNA (V3-V4) sequencing. A total of 285 bacterial genera were identified, with 196 genera in the HC group and 185 genera in the MS group. Alpha diversity by Chao1 index demonstrated decreased bacterial richness in the MS group compared with the $\mathrm{HC}$ group ( $p=0.020$; Fig 4A), aligning with our previous study showing less bacterial diversity in the gut of MS patients². Additionally, the MS and $\mathrm{HC}$ groups clustered separately on principal coordinate analysis of beta diversity using Bray-Curtis dissimilarity ( $p=0.004$; Fig 4B), indicating distinct microbiota profiles.

The five most prominent bacterial phyla across all the samples were Actinobacteria, Bacteroidetes, Firmicutes, Proteobacteria, and Verrucomicrobiota, accounting for 98.8\% of all identified bacteria (Fig 4C). Within the Bacteroidetes phylum, the Barnesiellaceae $\left(p_{a d j}=4.4 e-3\right)$ family and one of its genera, Barnesiella $\left(p_{a d j}=5.9 e-3\right)$, as well as the genus Odoribacter (Odoribacteraceae family; $\mathrm{p}_{\mathrm{adj}}=0.045$ ) were decreased in the MS group compared to HC (Supp 1 and Fig 4D). The other differentially abundant family, Eggerthellaceae (Actinobacteria phylum; $\mathrm{p}_{\mathrm{adj}}=6.3 \mathrm{e}-3$ ) and one of its genera, Eggerthella ( $\left.\mathrm{p}_{\mathrm{adj}}=2.4 \mathrm{e}-4\right)$, were overabundant in MS compared to HC (Supp 1 and Fig 4E). Within the Firmicutes phylum, an uncultured genus of the Oscillospiraceae family (Oscillospiraceae UCG 003) was depleted in the MS group compared to HC ( $\mathrm{p}_{\mathrm{adj}}=$ $0.045)$ while Blautia $\left(p_{a d j}=0.045\right)$ and Hungatella $\left(p_{a d j}=0.045\right)$ were increased in MS

(Fig 4E). We have previously also shown Prevotella to be significantly decreased in MS patients and beneficial in alleviating symptoms of EAE in mice through modulation of 
the gut microbiome ${ }^{2,28}$. In this study, the relative abundance of Prevotella was decreased in the MS group, though not significantly (padj=0.70; Supp 2). Similarly, Akkermansia was increased in MS but did not reach statistical significance $\left(p_{a d j}=0.73\right.$; Supp 2). Overall, the shift in the bacterial microbiome in MS patients in this study reveals a number of genera, several of which have already been described in MS microbiome literature.

\section{Correlation between Gut Mycobiome and Microbiome}

Comparing mycobiome changes with alterations in the bacterial microbiome of the samples show that with decreased bacterial richness comes increased fungal richness. The ITS2/16S ratio is increased in the MS group compared to the HC group $(p=0.040$; Fig 5A). Across all samples, bacterial and fungal richness were negatively correlated ( $R$ $=-0.28, p=0.042 ;$ Fig 5B).

Spearman correlation analysis reveals distinct fungal and bacterial interactions in MS and $\mathrm{HC}$ (Fig 5C). The microbial interactions in the $\mathrm{HC}$ group are dominated by negative correlations between several bacteria and six fungi. Meanwhile, the MS group exhibited more diverse relationships between the two kingdoms, with numerous positive associations appearing between various fungi and bacteria. MS patients with increased Blautia and Lactococcus in their stool had decreased Saccharomyces and increased Candida. Candida was also positively associated with Alistipes and Akkermansia. Additionally, Epicoccum and Cladosporium are negatively correlated with Prevotella. Penicillium is negatively correlated with Parasutterella and Holdemania and positive correlated with Terrisporobacter and Turicibacter. Aspergillus shares only positive correlations with multiple bacteria: Paludicola, an uncultured genus of the Oscillospiraceae family, and an uncultured genus of the Butyricicoccaceae family. 
This demonstrates a shift towards potentially more complex bacterial-fungal relationships in MS patients compared to $\mathrm{HC}$.

\section{Discussion}

This is the first study to characterize the gut fungal microbiome (mycobiome) profile of RRMS patients compared with healthy individuals and identify connections between the mycobiome and bacterial microbiome. Overall, the results of this study demonstrate that the mycobiota of MS patients differs from that of healthy individuals with enrichment of Candida, and Epicoccum, lower relative abundance of Saccharomyces, and increased ITS2/16S ratio in MS patients. This study highlights the important role of the understudied fungal microbiome in MS.

We observed that Ascomycota and Basidiomycota were the main phyla present in MS and HC. Ascomycota and Basidiomycota were negatively correlated with each other which had been previously shown in other autoimmune diseases such as IBD ${ }^{17}$ and $\mathrm{AS}^{16}$. MS patients had increased Basidiomycota compared to Ascomycota and an increase in BasidiomycotalAscomycota ratio, similar to the previously reported ratio in IBD ${ }^{17}$. While in AS patients, Ascomycota abundance was increased, and Basidiomycota abundance was decreased ${ }^{17}$. These related findings suggest that shifts in Ascomycota and Basidiomycota proportions might play a role in the pathogenesis of inflammatory diseases including MS. We detected two fungal genera, Candida and Epiccocum, with higher relative abundance, and one genus, Saccharomyces, with lower abundance in MS compared to HC. Although fungi belonging to Candida species are considered commensal microbes, this genus has also been linked with pathogenicity, especially in individuals with compromised immunity due to antibiotic usage, immunodeficiency, or barrier breach ${ }^{29}$. Interestingly, Candida species overgrowth had been reported in other autoimmune diseases such as IBD ${ }^{17,30}$. Thus, it 
is possible that bacterial dysbiosis and inflammatory conditions in the gut create favorable environments for expansion of Candida species which can then exacerbate inflammatory diseases such as MS and IBD through induction of pro-inflammatory response. In contrast to Candida, Saccharomyces, which is considered a beneficial fungus, has been shown to be decreased in $\mathrm{IBD}^{17},{ }^{31}$. Our findings showing a decreased abundance of Saccharomyces and increased abundance of Candida in MS is in concordance with previous findings in $\mathrm{IBD}^{17}$. Additionally, in colitis it has been observed that $S$. cerevisiae may exhibit regulatory effects on the host, notably by inducing higher IL-10 production from dendritic cells compared to Candida ${ }^{17}$. Moreover, colonic tissue of mice receiving $S$. cerevisiae ${ }^{31}$ showed increased expression of IL-10, suggesting either $S$. cerevisiae has anti-inflammatory potential or is poorly adapted to an inflammatory environment ${ }^{17}$. As $\mathrm{MS}$ is an inflammatory disease, gut mucosal inflammation might explain the decrease in Saccharomyces and increase in Candida.

Gut bacterial profiling in this study revealed an increased relative abundance of Hungatella, Eggerthella, and Blautia as well as decreased relative abundance of Odoribacter, Barnesiella, and an unidentified genus of Oscillospiraceae. These findings

align with previous MS microbiome studies showing increased relative abundance of Blautia and Eggerthella in $\mathrm{MS}^{2,}{ }^{3}$ and decreased relative abundance of Barnesiella ${ }^{1}$. Additionally, one study using a mouse model had shown increased Odoribacter in mice with relapsing-remitting $\mathrm{EAE}^{32}$. Previous studies have shown depletion of Prevotella and higher abundance of Akkermansia in MS patients ${ }^{1,2,33}$, and while we also observed similar trends, these did not reach statistical significance. A number of factors could have contributed to differences in the microbiome profiles of our study, including different 16S rRNA variable region-specific primers, a more recently developed analysis pipeline (ASV vs OTU-based taxonomic mapping), as well as the geographical origin of 
the samples ${ }^{34-36}$. Additionally, our female to male ratio of 5:1 in this study is higher than 3:1 $1^{1,2}$ and $2: 1^{6}$ in previous studies, which could contribute to differences.

In a healthy state, there is a symbiosis between bacteria, fungi, viruses, and the host. Similar to bacteria, commensal fungi have been shown to play a significant role in maintaining immune homeostasis, and perturbation of the healthy mycobiome can influence the local and peripheral immune systems directly or indirectly through modulation of bacterial populations ${ }^{37-39}$. Fungi may directly interact with the immune system in the gut and influence innate and systemic immune response. Intestinal fungi and their metabolic products may also leak out of the enteric luminal surfaces and activate immune cells ${ }^{13,40-42}$. Besides the direct effect of fungi on the host, relationships between fungi and bacteria might play a critical role in host immunity. Fungi and bacteria can compete for nutrients and adhesion sites and modulate the environment ${ }^{43}, 44$. Antibiotics have been linked to increasing growth of fungi in the human intestinal tract ${ }^{45}$. In the mouse model, an increased fungal abundance was observed after antibiotic treatment which reduced after antibiotic cessation, pointing towards a balancing mechanism between microbiota and mycobiota in the gut ${ }^{17,18}$. Decreased bacterial diversity favors the growth of certain fungal species, and certain fungi can switch from commensal to pathogenic phenotypes ${ }^{43}$. In this study, we observed decreased bacterial alpha diversity and increased fungal to bacterial richness in MS compared to $\mathrm{HC}$, as evident from the ITS2/16S ratio, suggesting that the MS gut might favor fungi at the expense of bacteria.

The microbial interactions in the $\mathrm{HC}$ group are dominated by negative correlations between several bacteria and fungi. However, our MS group exhibited more diverse relationships between the bacteria and fungi. Specifically, MS patients with increased Candida also had increased Alistipes, Akkermansia, and Lachnospiraceae GCA, and 
those with increased Saccharomyces also had increased Lactococcus, Blautia, and Romboutsia. It has been shown that in healthy mucosa, yeast (e.g., Candida albicans) is kept at low levels or excluded by the indigenous bacterial microbiota by the mechanism of colonization resistance which is not yet defined ${ }^{46}$. In fact, a previous study showed that germ-free mice are highly susceptible to Candida infection and antibiotics treatment leads to an expansion of fungal population ${ }^{18,47}$. Additionally, IBD patients with decreased abundance of Saccharomyces had decreased abundance of several bacteria that are depleted in IBD, such as Bifidobacterium, Blautia, Roseburia, and Ruminococcus, while unidentified Malasseziale followed an opposing pattern ${ }^{17}$. In AS patients, there is a positive corelation between abundance of Saccharomyces to Clostridium sensu stricto, Escherichia/Shigella, and Veillonella and negative correlation between abundance of Saccharomyces to Roseburia and Faecalibacterium ${ }^{16}$. Thus, our study along with previous studies suggests a role of fungal mycobiome in MS as well as other autoimmune diseases.

Functional profiling of the fungal communities in this study suggests modulation of fungal carbohydrate and amino acid pathways. More specifically, while the functional profile of the MS group mycobiome suggested decreased cellulose metabolism by the gut mycobiome (decreased cellobiohydrolase and endoglucanase), starch metabolism by amylase was suggested to be increased. Invertase, which was decreased in MS, can be produced by fungi, mainly Saccharomyces cerevisiae. It hydrolyzes sucrose into glucose and fructose and also has antibacterial and antioxidant properties. Sucrose provides a primary substrate for the generation of starch and cellulose $\mathrm{A}^{48}$. Both cellulose and starch are carbohydrates that can be metabolized into short-chain fatty acids (SCFAs) in the human gut. However, while the metabolism of starches has been shown to increase fecal butyrate levels ${ }^{49}$, metabolism of cellulose-related structures ${ }^{50}$ leads to 
increased gut propionate levels ${ }^{51}$.

Currently, there are limited tools for functional analysis of the mycobiome, and this study utilized a still growing database of fungal functional profiles. Moreover, functional profiling of fungi is difficult due to the difficulty of culturing fungi and highly varied, environment-dependent fungal trophic modes ${ }^{24}$. The quality of these profiles derived from ITS1/2 sequencing data will continue to improve as the field progresses and these databases grow. Alternatively, while more cost and time-intensive, whole genome sequencing of the mycobiome would provide more detailed data for more reliable functional analysis of the mycobiome.

We acknowledge that our small sample size is a limitation of the study. However, the subject recruitment was impacted due to the COVID-19 pandemic. We did not want to recruit new subjects as the study may have been confounded by COVID-19 infections. Due to small sample size, we were also not able to analyze the impact of different drug treatments on bacterial and fungal composition, therefore we cannot rule out the impact of treatment on the microbiome. Another limitation of this study was the different BMI distributions in the $\mathrm{HC}$ and MS groups. Interestingly, BMI was not significantly correlated to most of the differentially abundant bacteria or fungi except for Eggerthella. Nonetheless, future studies would benefit from larger sample sizes in order to better isolate the microbiota differences related to MS from those related to BMI and different treatment groups.

\section{Conclusion}

We observed that RRMS patients exhibit altered gut mycobiome along with altered gut microbiome. RRMS patients also showed variations in bacterial and fungal relationships with increased ratios of fungal/bacterial abundance and richness. Shifts in fungal functional profiles were also observed in RRMS patients compared to HC. Thus, our 
data suggest that the mycobiome may play an important role in the pathobiology of MS.

Whether changes in the mycobiome play a role in initiating MS pathophysiology or modulating its presentation, or whether the pathology of MS leads to changes in the fungal mycobiome remains an important question for future studies.

\section{References}

1. Jangi S, Gandhi R, Cox LM, et al. Alterations of the human gut microbiome in multiple sclerosis. Nat Commun. 2016 Jun 28;7:12015.

2. Chen J, Chia N, Kalari KR, et al. Multiple sclerosis patients have a distinct gut microbiota compared to healthy controls. Sci Rep. 2016 Jun 27;6:28484.

3. Miyake S, Kim S, Suda W, et al. Dysbiosis in the Gut Microbiota of Patients with Multiple Sclerosis, with a Striking Depletion of Species Belonging to Clostridia XIVa and IV Clusters.

PLoS One. 2015;10(9):e0137429.

4. Ochoa-Reparaz J, Kasper LH. Gut microbiome and the risk factors in central nervous system autoimmunity. FEBS Lett. 2014 Nov 17;588(22):4214-22.

5. $\quad$ Freedman SN, Shahi SK, Mangalam AK. The "Gut Feeling": Breaking Down the Role of Gut Microbiome in Multiple Sclerosis. Neurotherapeutics. 2018 Jan;15(1):109-25.

6. Cekanaviciute E, Yoo BB, Runia TF, et al. Gut bacteria from multiple sclerosis patients modulate human T cells and exacerbate symptoms in mouse models. Proc Natl Acad Sci U S A. 2017 Oct 3;114(40):10713-8.

7. Cosorich I, Dalla-Costa G, Sorini C, et al. High frequency of intestinal TH17 cells correlates with microbiota alterations and disease activity in multiple sclerosis. Sci Adv. 2017 Jul;3(7):e1700492.

8. Mangalam A, Yadav M, Yadav R. The emerging world of microbiome in autoimmune disorders: Opportunities and challenges. Indian Journal of Rheumatology. 2021 March 1, 2021;16(1):57-72.

9. Huseyin CE, O'Toole PW, Cotter PD, Scanlan PD. Forgotten fungi-the gut mycobiome in human health and disease. FEMS Microbiol Rev. 2017 Jul 1;41(4):479-511.

10. Qin J, Li R, Raes J, et al. A human gut microbial gene catalogue established by metagenomic sequencing. Nature. 2010 Mar 4;464(7285):59-65.

11. Arumugam M, Raes J, Pelletier E, et al. Enterotypes of the human gut microbiome. Nature. 2011 May 12;473(7346):174-80.

12. Yeung F, Chen YH, Lin JD, et al. Altered Immunity of Laboratory Mice in the Natural Environment Is Associated with Fungal Colonization. Cell Host Microbe. 2020 May 13;27(5):809-22 e6.

13. Wheeler ML, Limon JJ, Bar AS, et al. Immunological Consequences of Intestinal Fungal Dysbiosis. Cell Host Microbe. 2016 Jun 8;19(6):865-73.

14. Mestas J, Hughes CC. Of mice and not men: differences between mouse and human immunology. J Immunol. 2004 Mar 1;172(5):2731-8.

15. Al Bataineh MT, Dash NR, Lassen PB, et al. Revealing links between gut microbiome and its fungal community in Type 2 Diabetes Mellitus among Emirati subjects: A pilot study. Sci Rep. 2020 Jun 15;10(1):9624.

16. Li M, Dai B, Tang Y, et al. Altered Bacterial-Fungal Interkingdom Networks in the Guts of Ankylosing Spondylitis Patients. mSystems. 2019 Mar-Apr;4(2).

17. Sokol H, Leducq V, Aschard H, et al. Fungal microbiota dysbiosis in IBD. Gut. 2017 Jun;66(6):1039-48.

18. Dollive S, Chen YY, Grunberg S, et al. Fungi of the murine gut: episodic variationand proliferation during antibiotic treatment. PLoS One. 2013;8(8):e71806. 
19. Shahi SK, Zarei K, Guseva NV, Mangalam AK. Microbiota Analysis Using Two-step PCR and Next-generation 16S rRNA Gene Sequencing. J Vis Exp. 2019 Oct 15(152).

20. Op De Beeck M, Lievens B, Busschaert P, Declerck S, Vangronsveld J, Colpaert JV. Comparison and validation of some ITS primer pairs useful for fungal metabarcoding studies. PLoS One. 2014;9(6):e97629.

21. Callahan BJ, McMurdie PJ, Rosen MJ, Han AW, Johnson AJ, Holmes SP. DADA2: High-resolution sample inference from Illumina amplicon data. Nat Methods. 2016 Jul;13(7):581-3.

22. Nilsson $\mathrm{RH}$, Larsson $\mathrm{KH}$, Taylor AFS, et al. The UNITE database for molecular identification of fungi: handling dark taxa and parallel taxonomic classifications. Nucleic Acids Res. 2019 Jan 8;47(D1):D259-D64.

23. Quast C, Pruesse E, Yilmaz P, et al. The SILVA ribosomal RNA gene database project: improved data processing and web-based tools. Nucleic Acids Res. 2013 Jan;41(Database issue):D590-6.

24. Põlme S, Abarenkov K, Henrik Nilsson R, et al. FungalTraits: a user-friendly traits database of fungi and fungus-like stramenopiles. Fungal Diversity. 2020 2020/11/01;105(1):1-

16.

25. Jari Oksanen, F. Guillaume Blanchet, Michael Friendly, et al. vegan: Community Ecology Package. version 2.5-7 ed2020.

26. A. K. Ggpubr: 'Ggplot2’ Based Publication Ready Plots. Package Version 0.2 ed2018.

27. Kursa MB, Rudnicki WR. Feature Selection with the Boruta Package. 2010. 20102010 09-16;36(11):13.

28. Mangalam A, Shahi SK, Luckey D, et al. Human Gut-Derived Commensal Bacteria Suppress CNS Inflammatory and Demyelinating Disease. Cell Rep. 2017 Aug 8;20(6):1269-77.

29. Dongari-Bagtzoglou A, Fidel PL, Jr. The host cytokine responses and protective immunity in oropharyngeal candidiasis. J Dent Res. 2005 Nov;84(11):966-77.

30. Li J, Chen D, Yu B, et al. Fungi in Gastrointestinal Tracts of Human and Mice: from Community to Functions. Microb Ecol. 2018 May;75(4):821-9.

31. Jawhara S, Habib K, Maggiotto F, et al. Modulation of intestinal inflammation by yeasts and cell wall extracts: strain dependence and unexpected anti-inflammatory role of glucan fractions. PLoS One. 2012;7(7):e40648.

32. Gandy KAO, Zhang J, Nagarkatti P, Nagarkatti M. The role of gut microbiota in shaping the relapse-remitting and chronic-progressive forms of multiple sclerosis in mouse models. Sci Rep. 2019 May 6;9(1):6923.

33. Vallino A, Dos Santos A, Mathe CV, et al. Gut bacteria Akkermansia elicit a specific lgG response in CSF of patients with MS. Neurol Neuroimmunol Neuroinflamm. 2020 May;7(3).

34. Gupta VK, Paul S, Dutta C. Geography, Ethnicity or Subsistence-Specific Variations in Human Microbiome Composition and Diversity. Front Microbiol. 2017;8:1162.

35. Yatsunenko T, Rey FE, Manary MJ, et al. Human gut microbiome viewed across age and geography. Nature. 2012 May 9;486(7402):222-7.

36. Rehman A, Rausch P, Wang J, et al. Geographical patterns of the standing and active human gut microbiome in health and IBD. Gut. 2016 Feb;65(2):238-48.

37. Leonardi I, Li X, Semon A, et al. CX3CR1(+) mononuclear phagocytes control immunity to intestinal fungi. Science. 2018 Jan 12;359(6372):232-6.

38. Becker KL, Ifrim DC, Quintin J, Netea MG, van de Veerdonk FL. Antifungal innate immunity: recognition and inflammatory networks. Semin Immunopathol. 2015 Mar;37(2):10716.

39. Iliev ID, Funari VA, Taylor KD, et al. Interactions between commensal fungi and the Ctype lectin receptor Dectin-1 influence colitis. Science. 2012 Jun 8;336(6086):1314-7.

40. Wheeler ML, Limon JJ, Underhill DM. Immunity to Commensal Fungi: Detente and Disease. Annu Rev Pathol. 2017 Jan 24;12:359-85.

41. Zhang Z, Li J, Zheng W, et al. Peripheral Lymphoid Volume Expansion and Maintenance Are Controlled by Gut Microbiota via RALDH+ Dendritic Cells. Immunity. 2016 Feb 16;44(2):330-42.

42. Limon JJ, Skalski JH, Underhill DM. Commensal Fungi in Health and Disease. Cell Host 
Microbe. 2017 Aug 9;22(2):156-65.

43. Kruger W, Vielreicher S, Kapitan M, Jacobsen ID, Niemiec MJ. Fungal-Bacterial Interactions in Health and Disease. Pathogens. 2019 May 21;8(2).

44. Haddad JG, Jr., Boisseau V, Avioli LV. Phosphorus deprivation: the metabolism of vitamin D 3 and 25-hydroxycholecalciferol in rats. J Nutr. 1972 Feb;102(2):269-81.

45. Samonis G, Gikas A, Anaissie EJ, et al. Prospective evaluation of effects of broadspectrum antibiotics on gastrointestinal yeast colonization of humans. Antimicrob Agents Chemother. 1993 Jan;37(1):51-3.

46. Erb Downward JR, Falkowski NR, Mason KL, Muraglia R, Huffnagle GB. Modulation of post-antibiotic bacterial community reassembly and host response by Candida albicans. Sci Rep. 2013;3:2191.

47. Bertolini M, Ranjan A, Thompson A, et al. Candida albicans induces mucosalbacterial dysbiosis that promotes invasive infection. PLoS Pathog. 2019 Apr;15(4):e1007717.

48. Manoochehri H, Hosseini NF, Saidijam M, Taheri M, Rezaee H, Nouri F. A review on invertase: Its potentials and applications. Biocatalysis and Agricultural Biotechnology. 2020 2020/05/01/;25:101599.

49. McOrist AL, Miller RB, Bird AR, et al. Fecal butyrate levels vary widely among individuals but are usually increased by a diet high in resistant starch. J Nutr. 2011 May;141(5):883-9.

50. Behera BC, Sethi BK, Mishra RR, Dutta SK, Thatoi HN. Microbial cellulases - Diversity \& biotechnology with reference to mangrove environment: A review. J Genet Eng Biotechnol. 2017 Jun;15(1):197-210.

51. Grootaert C, Van den Abbeele P, Marzorati M, et al. Comparison of prebiotic effects of arabinoxylan oligosaccharides and inulin in a simulator of the human intestinal microbial ecosystem. FEMS Microbiol Ecol. 2009 Aug;69(2):231-42. 


\section{Study Funding}

This work was supported by a grant from Schwab Foundation Margaret Heppelmann and Michael Wacek, departmental fund, NIH R01 grant 1R01Al137075-01, and VA Merit Award 1101CX002212 to Ashutosh Mangalam.

Meeta Yadav is supported by NRSA T90 training grant 5T90DE023520. Rachel Shrode is funded by the University of lowa's Informatics Fellowship from the Informatics Graduate Program. Soham Ali is supported by the Emory Warner Medical Student Research Fellowship from the University of lowa Department of Pathology.

\section{Disclosure}

AM is one of the inventors of a technology claiming the use of Prevotella histicola to treat autoimmune diseases. AM received royalties from Mayo Clinic (paid by Evelo Biosciences). MY, SA, RS, SS, SJ, JH, SC, HO, NG, MP, CC, KW, TC, JK declare no commercial or financial relationships that could be a potential conflict of interest. 

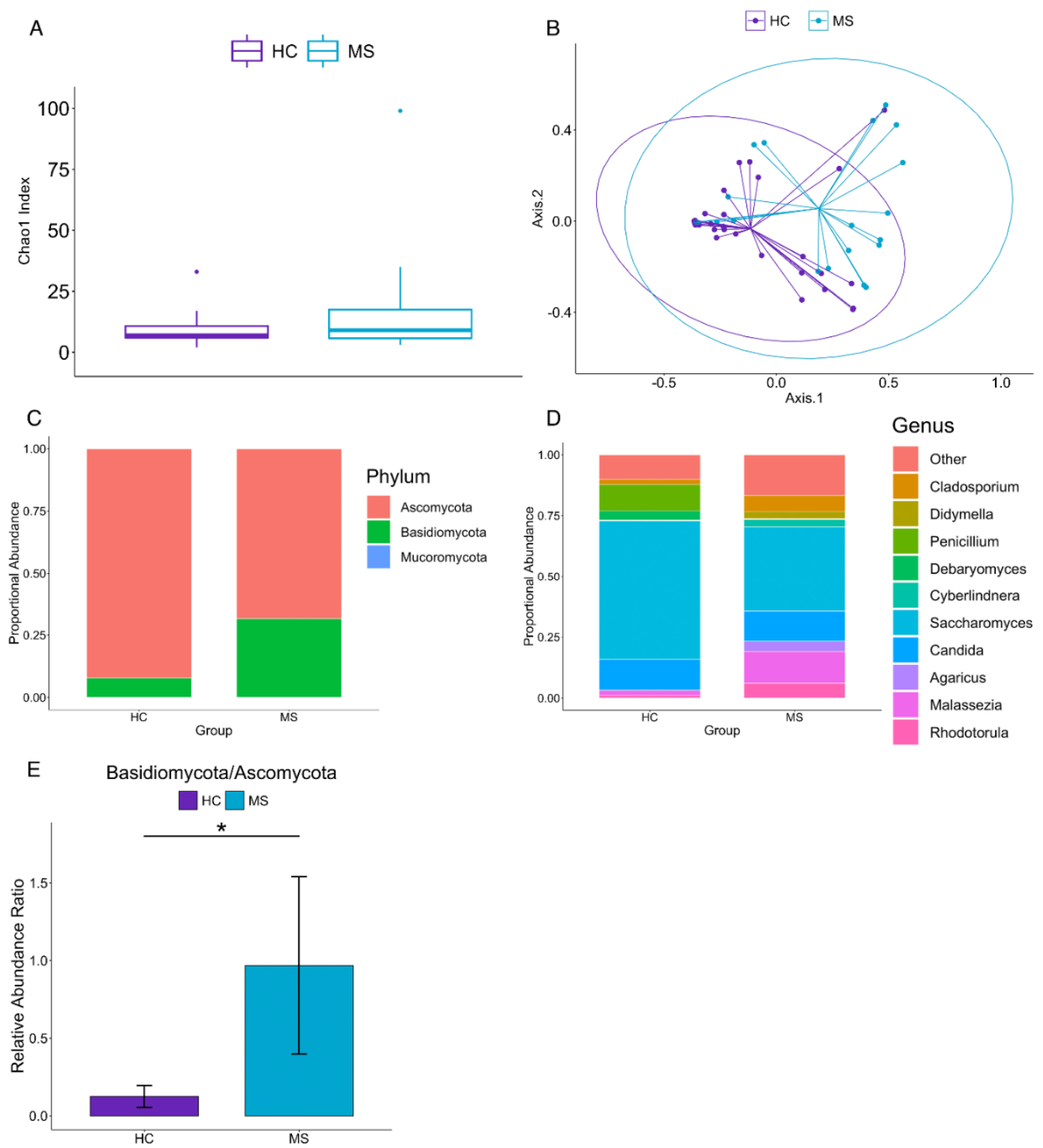

Figure 1. Fungal microbiota of RRMS patients is different from healthy controls $(A)$

Fungal ASV richness estimated by Chao1 index in MS and HC. (B) Principal coordinate analysis of Bray-Curtis dissimilarity of $\mathrm{HC}$ and MS shows that the mycobiome of $\mathrm{HC}$ and MS are distinct (PERMANOVA: $p=0.004$ ). Ellipses correspond to $95 \%$ confidence intervals around the centroids for each group. (C) Bar plot showing the relative abundances of fungal phyla. Basidiomycota was increased and Ascomycota was decreased in MS compared to HC. (D) Bar plot showing the top 10 fungal genera in HC and MS (determined by average relative abundance across all samples). The top 10 genera account for $85.5 \%$ of all identified fungal genera. (E) Basidiomycota/Ascomycota ratio is significantly increased in MS $(p=0.0053)$. 

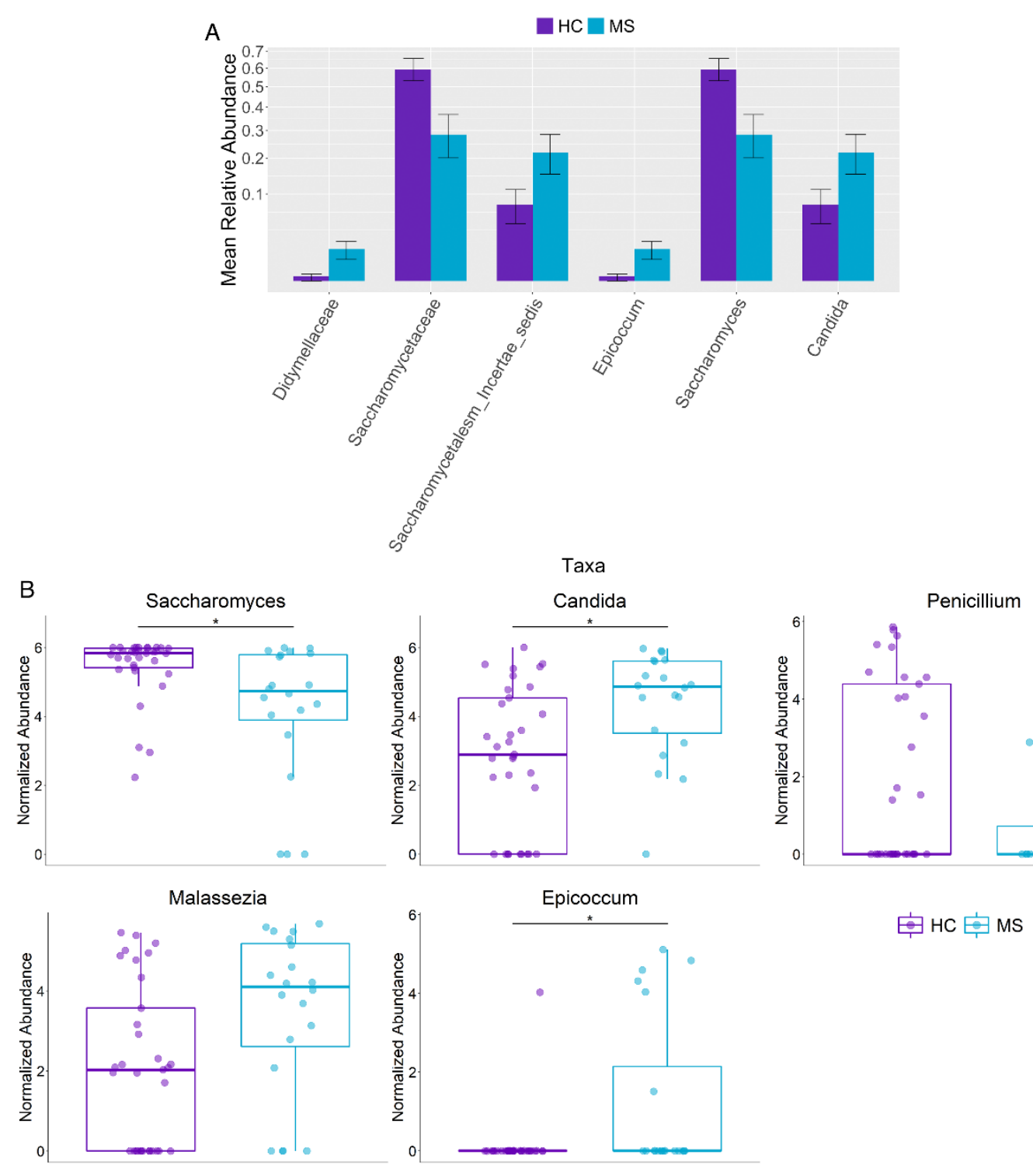

C

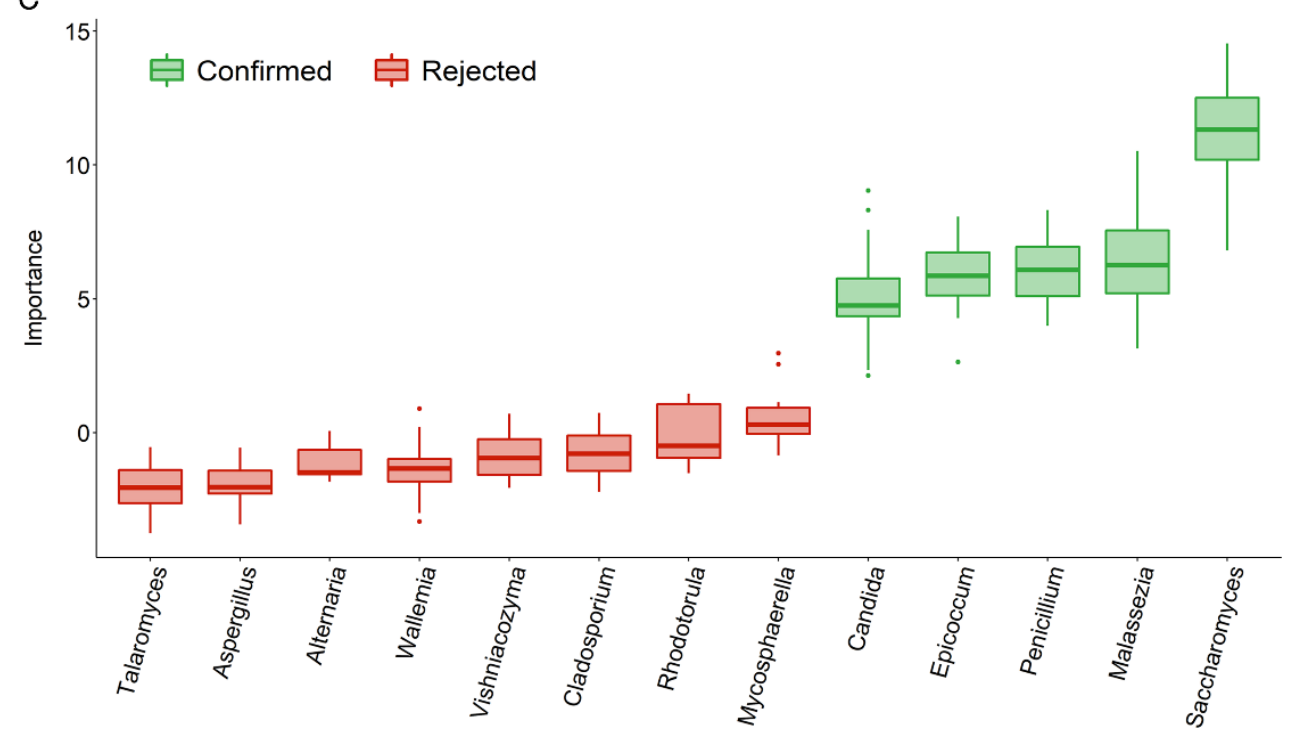

Figure 2. Differentially abundant fungal genera in RRMS versus healthy controls 
(A) Bar plot showing relative abundances of differentially abundant taxa $(p<0.05)$ at the phylum, family, and genus level. (B) Differentially abundant fungal genera in MS and $\mathrm{HC}$ using Wilcoxon signed rank test and adjusted for multiple comparisons with the Benjamini-Hochberg method at a significance level of 0.05. Candida, Epicoccum, and Malassezia are increased in MS compared to HC. Saccharomyces is decreased in MS compared to HC. Penicillium was identified in random forest analysis as a significant feature and was decreased in MS, though the Wilcoxon test did not reach statistical significance after adjusting for multiple comparisons. Abundance values are sum-scaled to 1 million and generalized log-transformed. The * symbol indicates $p$ value $<0.05$. (C) Importance of features determined by random forest and tested for significance with the Boruta algorithm at a significance level of 0.01 .
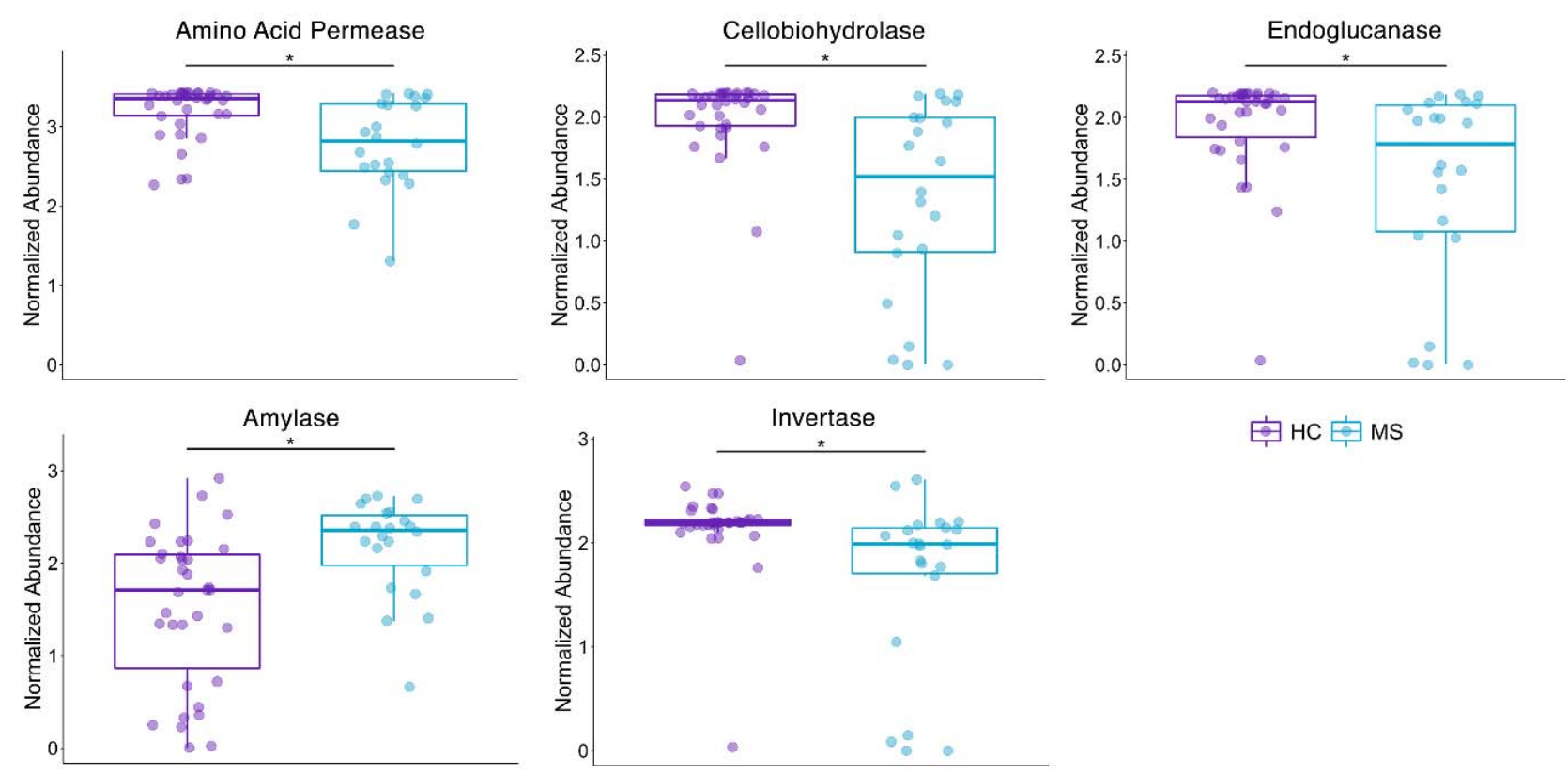

审 $\mathrm{HC}$ 审MS

Figure 3. Functional Profile of Gut Mycobiome in MS Patients Differentially enriched fungal functions using Wilcoxon signed rank test and adjusted for multiple comparisons with the Benjamini-Hochberg method at a significance level of 0.05. Amino acid permease, cellobiohydrolase, endoglucanase, and invertase are decreased in MS compared to $\mathrm{HC}$. Amylase is increased in MS compared to $\mathrm{HC}$. Abundance values are sum-scaled to 1 million and generalized log-transformed 

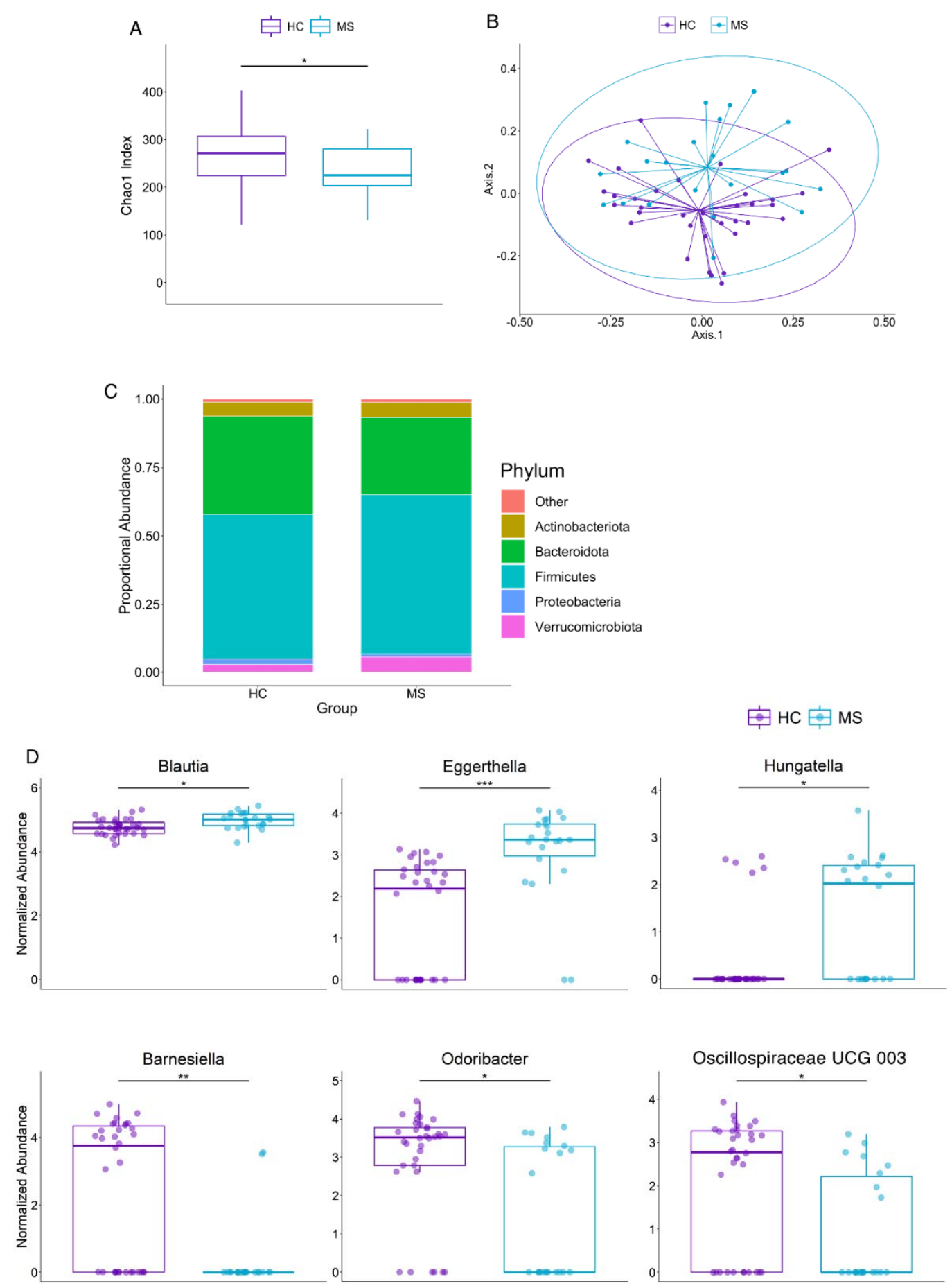

Figure 4. Gut Bacterial Microbiome of RRMS patients is different from healthy $(A)$ Bacterial ASV richness estimated by Chao1 index showing decreased bacterial richness in MS $(p=0.020)$. (B) Principal coordinate analysis of Bray-Curtis dissimilarity of $\mathrm{HC}$ and $\mathrm{MS}$ showing that the microbiome of $\mathrm{HC}$ and $\mathrm{MS}$ are distinct (PERMANOVA: $\mathrm{p}=0.004$ ). Ellipses are visual and do not correspond to any statistical analysis. (C) Abundance of bacteria at phylum level. (D) Differentially abundant bacteria between $\mathrm{HC}$ and MS using Wilcoxon signed rank test and adjusted for multiple comparisons with the Benjamini-Hochberg method at a significance level of 0.05 . Abundance values are sum-scaled to 1 million and generalized log-transformed. The ${ }^{* * *}$, and ${ }^{* * *}$ symbol indicates $p$-values of $<0.05,<0.01,<0.001$, respectively. 
A

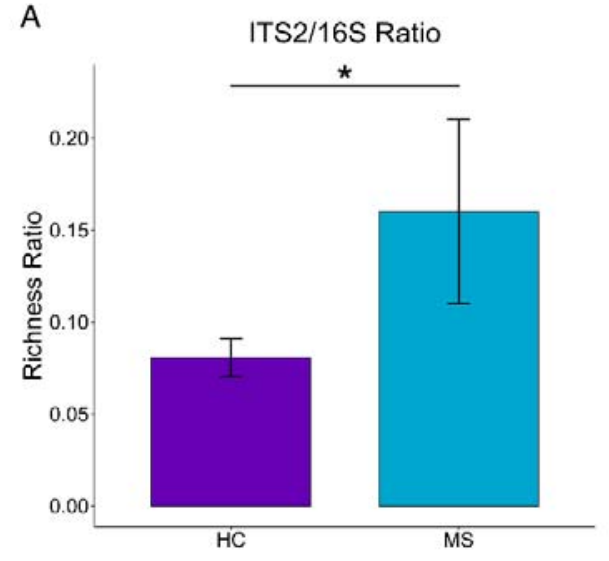

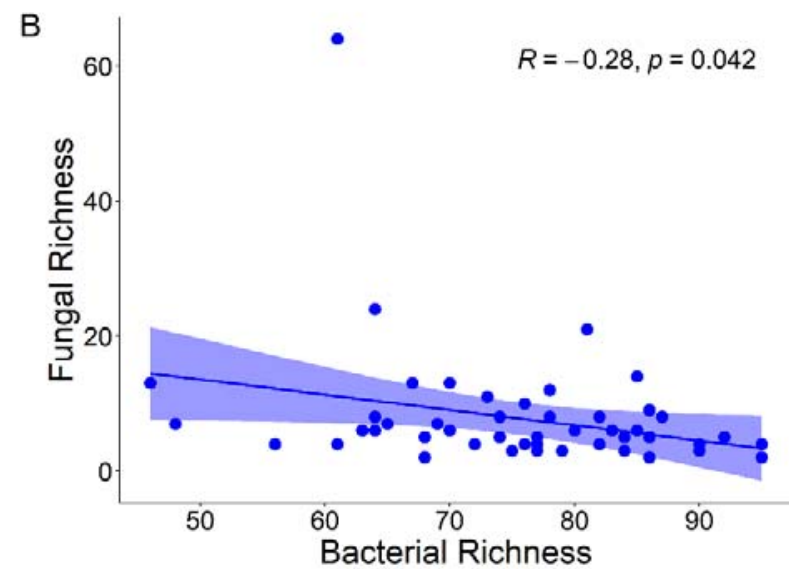

C

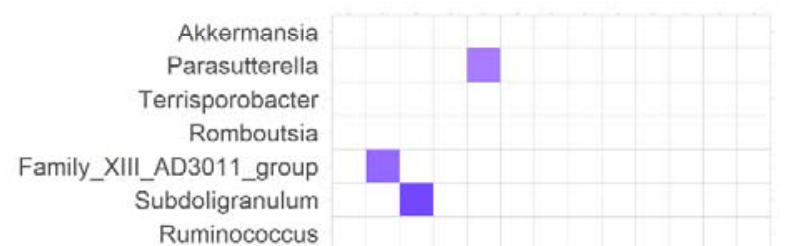

Paludicola

Oscillospiraceae UCG_005

Oscillospiraceae UCG_003

Oscillospiraceae UCG_002

Lachnospiraceae NK4A214_group

Intestinimonas

Colidextribacter

Butyricicoccaceae UCG_009

Monoglobus

Tuzzerella

Marvinbryantia

Lachnospiraceae_ND3007_group

Lachnospiraceae_FCS020_group

Hungatella

Lachnospiraceae GCA_900066575

Fusicatenibacter

Eisenbergiella

Coprococcus

Blautia

Christensenellaceae_R_7_group

Lactococcus

Turicibacter

Holdemania

Erysipelotrichaceae_UCG_003

Desulfovibrio

Parabacteroides

Alistipes

Prevotella

Butyricimonas

Collinsella

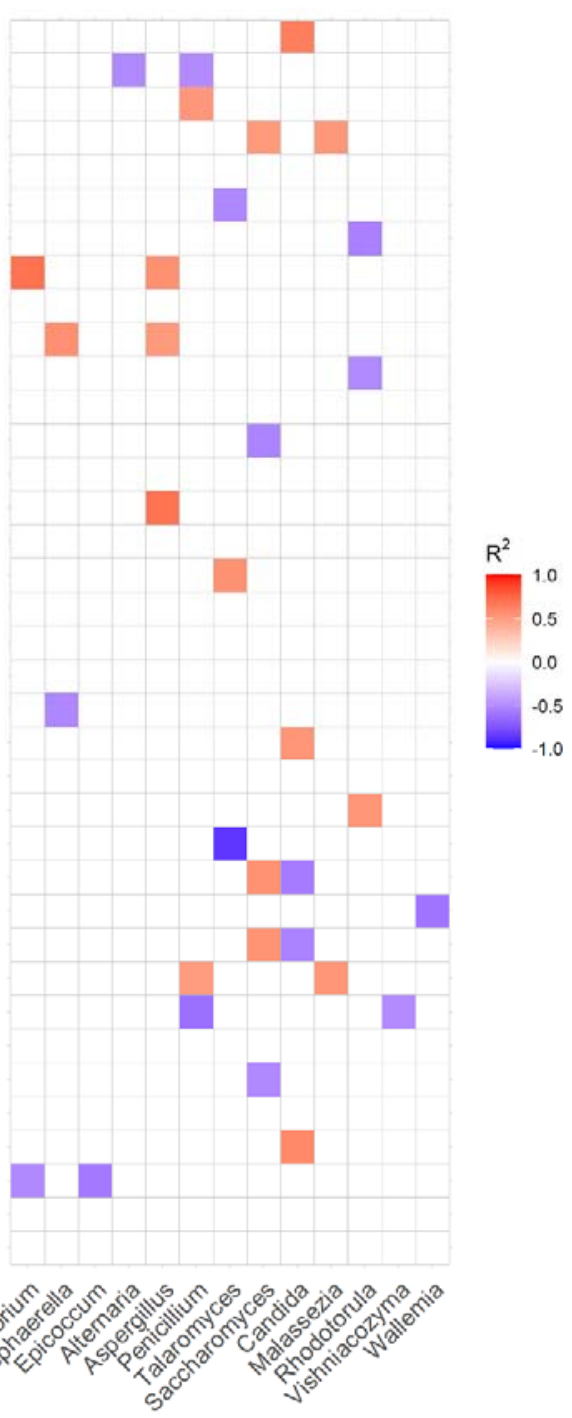

Figure 5. Correlation between Gut Mycobiome and Microbiome (A) Ratio of ITS2 to $16 S$ compared between groups. (B) Linear regression of fungal and bacterial richness shows a negative correlation (Spearman's $R=-0.28, p=0.042$ ). (C) Correlation matrix between bacteria and fungi using Spearman correlation. Values range from -1 to 1 with positive values as orange and red and negative values as purple and blue $(-1 \leq R$ $\leq 1)$. Only statistically significant correlations $(p<0.05)$ are shown. 